\title{
Pressure in generalized parton distributions and distribution amplitudes
}

\section{O.V. Teryaev*}

JINR, 141980, Dubna, Russia

E-mail: teryaev@theor.jinr.ru

The recent report on pressure distribution in proton supports its similarity to macroscopic stable object. This is valid for other particles and, surprisingly enough, for photons The general properties of Generalized Parton Distributions and Generalized Distribution Amplitudes are discurssed. There are indications that Extended Equivalence Principle wich was suggested earlier to explain the Anomalous Gravitomagnetic Moments of quarks and gluons may be generalized to their pressures and shear forces. The latter may be studied in the inclusive hard processes of tensor polarized gluons.

23rd International Spin Physics Symposium - SPIN2018 -

10-14 September, 2018

Ferrara, Italy

\footnotetext{
* Speaker.
} 


\section{Introduction}

The recent experimental investigation [1] of the pressure in proton exploring the original brilliant suggestion [2] and its further development [3, 4] resulted for the first time in the impressive picture of the pressure distribution inside proton similar to that in the macroscopic object like star, manifesting the Nature conformity at the vary different scales.

It is very interesting that this stability property holds for all the known hadrons and their models [3] and, moreover, for photons [5]. Although consideration of the latter as a static spherically symmetric object which was analyzed originally [3] is problematic (although being slightly offshell, photon is space-like, so its "rest mass" is, naively, imaginary), the consideration of relevant subtraction, considered in the following sections, is still possible.

Here I discuss the implications and possible generalizations of these results for various aspects of hadron structure.

\section{Stability and global properties of GPDs}

The extraction of pressure is essentially based on the dispersion relations for DVCS amplitude $[6,7]$ resulting, at the leading order, in the following "holographic" sum rule [6],

$$
\int_{-1}^{1} d x \frac{H(x, \xi)-H(x, x)}{x-\xi+i \varepsilon}=\int_{-1}^{1} d z \frac{D(z)}{z-1}
$$

where D-term enters. The stability requires it to be negative which is ptrovided by the $H$ being the decreasing function of its second argument (skewness):

$$
\left(\xi_{1}>\xi_{2}\right) \rightarrow\left(H\left(x, \xi_{1}\right)<H\left(x . \xi_{2}\right)\right) .
$$

This is yet another example of global constraint, similar to positivity ones[8]. It is of special interest to recall the convexity of parton distributions preserved by evolution [8].

\section{Stability and GDAs}

The gravitational formfactors [9], related to pressure, may be also considered in the time-like region, and this opportunity was recently explored to get the relevant information for pion [10]. The holographic sum rule may be also applied in that channel with the same r.h.s. (provided that both $s$ and $t$ variables are considered to be negligibly small, while the more accurate description is provided by the corresponding dispersion relation [11]), so that one can get (c.f. [12]) for $\xi>1$ :

$$
\int_{-1}^{1} d x\left[\frac{\Phi(x, 1 / \xi)}{x-1}-\frac{H(x, x)}{x-\xi}\right] \cdot=\int_{-1}^{1} d z \frac{D(z)}{z-1},
$$

where GDA is related to $H$ for $\xi>1$ [13]. As a result, the higher harmonics in angular distributions, corresponding to large powers of $1 / \xi$, are related to higher moments of $H(x, x)$ [12]. Concerning stability, it is automatically preserved by negative GDA, while positive one should be bounded from above. 


\section{Shear forces and tensor polarisation}

The natural way to get the traceless part of energy-momentum tensor matrix element is provided by tensor polarisation. As the latter is P-even, one may consider forward matrix elements, revealed in inclusive processes [14]. The DIS data are compatible [14] with the equivalence principle valid separately for quarks and gluons, like it was originally suggested for anomalous gravitomagnetic moments [9] and was recently discovered for their pressure [15].

To check this picture one may use future studies of DIS at JLab and of Drell-Yan process with tensor polarized deuterons [16]. This opportunity, in addition to KEK, may be provided by the NICA collider ar JINR [17] ${ }^{1}$.

Let me also stress here, that tensor polarised parton distribution may be also measured in any hard process with the relevant combination of deuteron polarisations, in particular, for large $p_{T}$ pions production, providing much better statistics. To my best knowledge, such opportunity, albeit straightforward, was not yet mentioned explicitely and deserves to be explored further.

\section{Conclusions}

The stability of hadrons and photons may be used to put the cosntraints for their non-perturbative matrix elements. The notion of equivalence principle may be extended to valid separately for quarks and gluons, including their gravitomagnetic moments, pressure and shear forces.

I am indebted to Igor Anikin, Volker Burkert, Ilnur Gabdrakhmanov, Francois-Xavier Girod, Shunzo Kumano, Cedrik Lorcé, Dieter Müller, Barbara Pasquini, Maxim Polyakov, Peter Schweitzer, Qin-Tao Song for useful discussions.

I would like to dedicate this paper to my teacher, Anatoli Vasil'evich Efremov, on occasion on his 85 th Birthday.

\section{References}

[1] V. D. Burkert, L. Elouadrhiri and F. X. Girod, Nature 557 (2018) no.7705, 396. doi:10.1038/s41586-018-0060-Z

[2] M. V. Polyakov, Phys. Lett. B 555 (2003) 57 doi:10.1016/S0370-2693(03)00036-4 [hep-ph/0210165].

[3] M. V. Polyakov and P. Schweitzer, Int. J. Mod. Phys. A 33 (2018) no.26, 1830025 doi:10.1142/S0217751X18300259 [arXiv:1805.06596 [hep-ph]].

[4] C. LorcÃ̂l', H. Moutarde and A. P. TrawiÅĎski, arXiv:1810.09837 [hep-ph].

[5] I. R. Gabdrakhmanov and O. V. Teryaev, Phys. Lett. B 716 (2012) 417 doi:10.1016/j.physletb.2012.08.041 [arXiv:1204.6471 [hep-ph]].

[6] O. V. Teryaev, hep-ph/0510031.

[7] I. V. Anikin and O. V. Teryaev, Phys. Rev. D 76 (2007) 056007 doi:10.1103/PhysRevD.76.056007 [arXiv:0704.2185 [hep-ph]].

\footnotetext{
${ }^{1}$ Complementary probes are provided by vector mesons [18].
} 
[8] X. Artru, M. Elchikh, J. M. Richard, J. Soffer and O. V. Teryaev, Phys. Rept. 470 (2009) 1 doi:10.1016/j.physrep.2008.09.004 [arXiv:0802.0164 [hep-ph]].

[9] O. V. Teryaev, Front. Phys. (Beijing) 11 (2016) no.5, 111207. doi:10.1007/s11467-016-0573-6; hep-ph/9904376.

[10] S. Kumano, Q. T. Song and O. V. Teryaev, Phys. Rev. D 97 (2018) no.1, 014020 doi:10.1103/PhysRevD.97.014020 [arXiv:1711.08088 [hep-ph]].

[11] B. Pasquini, M. V. Polyakov and M. Vanderhaeghen, Phys. Lett. B 739 (2014) 133 doi:10.1016/j.physletb.2014.10.047 [arXiv:1407.5960 [hep-ph]].

[12] O. Teryaev, PoS DIS 2010 (2010) 250. doi:10.22323/1.106.0250

[13] O. V. Teryaev, Phys. Lett. B 510 (2001) 125 doi:10.1016/S0370-2693(01)00564-0 [hep-ph/0102303].

[14] O. V. Teryaev, Mod. Phys. Lett. A 24 (2009) 2831. doi:10.1142/S0217732309001042

[15] M. V. Polyakov and H. D. Son, JHEP 1809 (2018) 156 [JHEP 2018 (2020) 156] doi:10.1007/JHEP09(2018)156 [arXiv:1808.00155 [hep-ph]].

[16] S. Kumano and Q. T. Song, arXiv:1702.01477 [nucl-th].

[17] I. A. Savin et al., EPJ Web Conf. 85 (2015) 02039 doi:10.1051/epjconf/20158502039 [arXiv:1408.3959 [hep-ex]].

[18] O. V. Teryaev, AIP Conf. Proc. 915 (2007) 260 doi:10.1063/1.2750776 [hep-ph/0612205]. 